\title{
Review: counselling and education may improve outcomes in caregivers of patients with stroke
}

Visser-Meily A, van Heugten C, Post M, et al. Intervention studies for caregivers of stroke survivors: a critical review. Patient Educ Couns 2005; 56:257-67.

What is the effectiveness of different types of interventions for caregivers of patients with stroke?

\section{METHODS}

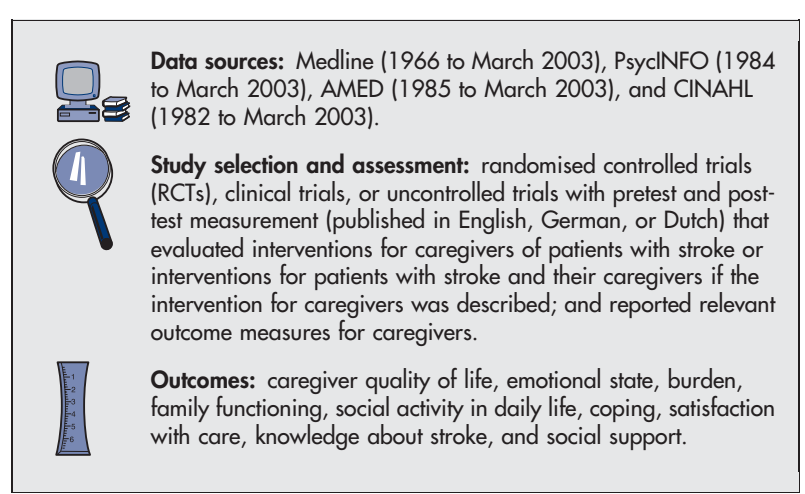

\section{MAIN RESULTS}

18 RCTs and 4 non-randomised studies (of which 6 were of interventions for caregivers only) met the selection criteria. 4 main intervention approaches were identified. (1) Providing specialist services (10 RCTs). The interventions were directed at improving and facilitating discharge from hospital. Typically, a stroke nurse or stroke organiser visited to give information about health services and therapies available in the community and to give advice and emotional support to both stroke patients and their caregivers. 3 of 10 RCTs found significant overall improvements for caregivers. Positive effects included improvements in emotional state and satisfaction with care (1 RCT), increases in knowledge (1 RCT), and improvements in health related quality of life and social activities of daily life ( 1 RCT). In 6 RCTs, the intervention and control groups did not differ for caregiver outcomes. In 2 RCTs, however, negative caregiver outcomes were reported in the intervention groups: a worse state of general health among caregivers in 1 RCT and dissatisfaction with provision of information coupled with a greater caregiver burden in the other.

(2) (Psycho)education (5 RCTs). Education was directed at gaining general knowledge about stroke or more specific knowledge about cognitive aspects. 3 of 5 RCTs reported significant improvements for caregivers. Positive effects included an increase in knowledge about stroke (1 RCT) and improvements in mental health (1 RCT). I RCT reported an increase in knowledge but deterioration in social functioning in the intervention group.

For correspondence: Dr A Visser-Meily, Rehabilitation Centre De Hoogstraat, Utrecht, The Netherlands. a.visser@dehoogstraat.nl

Source of funding: The Netherlands Organisation for Health Research and Development.
(3) Counselling (4 RCTs). Counselling elements included learning of goal setting, problem solving, and coping strategies. 3 of 4 RCTs found significant improvements for caregivers. Positive effects included facilitating and maintaining adaptive changes in family function (l RCT); improvements in problem solving skills and measures of vitality, greater caregiver preparedness, and less depression (1 RCT); and increases in knowledge about care, use of active coping strategies, and seeking social support (1 RCT).

(4) Peer support. No RCTs examined peer support in interventions such as stroke clubs.

\section{CONCLUSION}

Providing specialist services, (psycho)education, and counselling may improve outcomes in caregivers of patients with stroke.

\section{Commentary}

B efore the review by Visser-Meily ef al, the systematic review by Forster et al included 9 completed trials and 8 ongoing trials. 1 Although Forster et al found some evidence that information combined with educational sessions improved knowledge and was more effective than information provision alone, the conclusions about the effectiveness of information provision were not definitive because of low trial quality and variability among outcome measures. ${ }^{1}$

The review by Visser-Meily et al appears to have similar challenges in drawing firm conclusions about the effectiveness of interventions for caregivers of stroke survivors, although it highlights some approaches that may deliver benefits. To date, RCTs of specific stroke interventions have not adequately assessed the effects of caregiver characteristics, demographics, and physical health on caregiver intervention outcomes. ${ }^{2}$ Furthermore, optimal timing in the stroke recovery process, frequency, and duration of caregiver interventions have not been rigorously explored. ${ }^{3}$

Studies indicate that the effects of stroke on caregivers can be complex and multidimensional, affecting social networks and activities, roles, independence, and self identity. ${ }^{4}$ Nurses are uniquely positioned to support family caregivers and identify their (caregivers') most relevant individualised needs and tailor interventions appropriately. ${ }^{4}$

Nancy Boaro, RN, MN, CNN(c) Karima Velii, RN, MScN, AOCN

Toronto Rehabilitation Institute Toronto, Ontario, Canada

1 Forster A, Smith J, Young J, et al. Information provision for stroke patients and their caregivers. Cochrane Database Syst Rev 2001;(3):CD001919.

2 Han B, Haley WE. Family caregiving for patients with stroke. Review and analysis. Stroke 1999;30:1478-85.

3 Dowswell G, Lawler J, Dowswell T, et al. Investigating recovery from stroke: a qualitative study. J Clin Nurs 2000;9:507-15.

4 Bakas T, Austin JK, Okonkwo KF, et al. Needs, concerns, strategies, and advice of stroke caregivers the first 6 months after discharge. J Neurosci Nurs 2002;34:242-51. 